\title{
Verdad e incertidumbre en el marco del conflicto en Colombia: una mirada a los sistemas de información como prácticas de memoria ${ }^{1}$
}

\author{
Maria Fernanda Olarte-Sierra ${ }^{2}$ \\ Departamento de Diseño \\ Universidad de los Andes, Bogotá, Colombia ${ }^{3}$ \\ mf.olarte35@uniandes.edu.co \\ Adriana Diaz del Castillo H. ${ }^{4}$ \\ CESTA- investigación independiente \\ adiazdelc@gmail.com \\ Natalia Pulido Ronchaquira ${ }^{5}$ \\ Departamento de Antropología \\ Universidad de los Andes, Bogotá, Colombia ${ }^{6}$ \\ nataliapulidor@gmail.com \\ Nathalia Cabrera Villota ${ }^{7}$ \\ Departamento de Antropología \\ Universidad de los Andes, Bogotá, Colombia \\ cabreravillotanathalia@gmail.com \\ Roberto Suárez Montañes ${ }^{8}$ \\ Departamento de Antropología \\ Universidad de los Andes, Bogotá, Colombia ${ }^{9}$ \\ rsuarez@uniandes.edu.co \\ Recibido: 8 de noviembre de 2013 \\ Aceptado: 4 de febrero de 2014
}

Articulo de investigación científica resultado del proyecto de investigación "Sistemas de información en el marco de Justicia y Paz: ¿Medios para reparar la nación y escribir historia?” financiado por la convocatoria interdepartamental de la Universidad de los Andes en 2011.

2 Antropóloga de la Universidad de los Andes, maestra en Antropología médica. Doctora en Ciencias Sociales de la Universidad de Ámsterdam. Investigación post-doctoral en Antropología Social en la Universidad de Manchester.

3 Profesora asistente. Departamento de Diseño.

4 Magíster en Antropología médica de la Universidad de Ámsterdam y pregrado en medicina de la Universidad Nacional de Colombia.

5 Antropóloga de la Universidad de los Andes.

6 Estudiante de Maestría en Antropología Social de la Universidad de los Andes.

7 Estudiante de Antropología de la Universidad de los Andes

8 Especialista en antropologia médica. Licenciado en Anthropologia y Sociología de la Universidad de Lausanne. Doctor en Ciencias de la Educación de la Universidad de Ginebra, Suiza, Facultad de Psicología y Ciencias de la Educación (FPSE).

9 Profesor asociado, Departamento de Antropologia. 


\title{
Verdad e incertidumbre en el marco del conflicto en Colombia: una mirada a los sistemas de información como prácticas de memoria
}

\section{Resumen}

Desde los estudios sociales de ciencia y tecnología y basados en material etnográfico, analizamos el sistema de información disponible para la búsqueda, identificación y entrega de restos humanos en el conflicto colombiano. Entendemos que en este sistema confluyen prácticas de memoria con el poder de re-configurar el pasado (y el presente) a través de procesos de selección y exclusión enmarcados en prácticas científicas con carácter de verdad. Reconstruimos la trayectoria de producción de información e identificamos los filtros que operan al clasificarla y analizarla bajo estándares científicos. Seguimos los rastros que va dejando esta información excluida y que tienen el potencial de ser rescatados. Nos preguntamos por la memoria que se construye a partir de estos filtros y rastros. Argumentamos que es fundamental buscar que el sistema pueda abrir paso a la incertidumbre y contribuir asi a la producción de una memoria colectiva del conflicto que sea múltiple y matizada.

Palabras clave: prácticas de memoria; incertidumbre; sistema de información; conflicto; Colombia

\section{Truth and Uncertainty in the Framework of the Colombian Conflict: $A$ view to the Information Systems as Memory Practices}

\begin{abstract}
We analyzed the information system available for the search, identification and delivery of human remains in the Colombian conflict, based on the social studies on science and technology, and on ethnographic material. We understand that in this system the convergence of memory practices with the power to reconfigure past (and present) by means of selection and exclusion processes framed in scientific practices with character of truth is present. We reconstruct the path for the production of information and identify the filters that work when classifying and analyzing it under scientific standards. We follow the trails that the excluded information leaves and that may be potentially rescued. We question ourselves about the memory built from those filters and traces. We argument that it is vital striving to have the system open the way for uncertainty, so it contributes to the production of a collective memory of the conflict that is multiple and thorough.
\end{abstract}

Keywords: memory practices; uncertainty; information system; conflict; Colombia

\section{Verdade e incerteza no marco do conflito na Colômbia: um olhar aos sistemas de informação como práticas de memória}

\section{Resumo}

Desde os estudos sociais de ciência e tecnologia e baseadas no material etnográfico, analisamos o sistema de informação disponível para busca, identificação e entrega de restos mortais no conflito colombiano. Entendemos que neste sistema confluem práticas de memória com o poder de reconfigurar o passado (e o presente) através de processos de seleção e exclusão enquadrados em práticas científicas com carácter de verdade. Reconstruimos a trajetória de produção de informação e identificamos os filtros que operam ao classificála e analisá-la sob padrões científicos. Seguimos as trilhas deixadas por essa informação excluída e que tem potencial de ser resgatados. Questionamo-nos pela memória que é construída a partir destes filtros e trilhas. Argumentamos que é fundamental procurar o sistema puder abrir passo à incerteza e contribuir assim na produção de uma memória coletiva do conflito que seja múltipla e matizada.

Palavras-chave: práticas de memoria; incerteza; sistema de informação; conflito; Colômbia 
Lo que observamos no es la naturaleza en sí misma, sino la naturaleza expuesta a nuestro método de indagación.

Heisenberg (Law y Urry, 2004, p. 395)

\section{Introducción}

Experiencias en países como Guatemala, Argentina, Sudáfrica, Bosnia y Serbia, entre otros, evidencian la relevancia de los sistemas de información en procesos de paz para recopilar, almacenar y circular información que documente de manera confiable las violaciones a los derechos humanos. Es a través de ellos que se busca establecer el número de víctimas, identificar perpetradores y zonas geográficas afectadas e individualizar crímenes; en otras palabras, establecer "quién le hizo qué a quién" (Ball, Spirer, H. y Spirer, L., 2000).

Por lo tanto, los sistemas de información resultan centrales para los procesos de construcción de memoria colectiva (www.huridocs. org; Ball et al., 2000; Steinberg, Height, Mosher y Bampton, 2006; Knuth, 2006). Son lo que Jelin (2001) denomina la dimensión pasiva de la memoria, dado que al igual que los recuerdos y los registros almacenados en diversos lugares, son huellas del pasado. La construcción de memoria implica actividades llevadas a cabo para recuperar ciertos aspectos del pasado. Estas actividades incluyen recolectar y analizar datos con base en preguntas coyunturales del presente en contextos específicos y realizadas por actores particulares.

Hablamos de memoria colectiva entendiendo que la memoria es tanto individual como social y es un elemento fundamental y constitutivo de la identidad de personas y de grupos (Halbwachs y Namer, 1994; Groppo, 2002; Jelin, 2001). No se trata de asumir la memoria colectiva como un hecho dado sino como un conjunto de memorias entretejidas -compartidas algunas y debatidas otras- y sobre todo, entendemos que la construcción de memoria colectiva es un acto que responde a una politica particular de construcción de identidad (de una identidad colectiva particular). (Groppo, 2002; Jelin, 2001).

Ahora bien, los procesos de construcción de memoria son eminentemente selectivos: se rescatan unos aspectos específicos del pasado y se dejan de lado otros, no solo porque no podemos recordarlo todo, sino porque al construir memoria se construye un tipo particular de 
pasado en el que unos hechos se priorizan sobre otros. En esta medida, toda política de memoria tiene implícita una política de olvido (Groppo, 2002; Lvovich, 2002; Jelin, 2001). La memoria colectiva es reconstruida y resignificada de manera permanente, contingente y política en una disputa constante sobre el sentido y el significado del pasado desde el presente. Es así como, en términos de construcción de memoria, "la cuestión no es tanto sobre los hechos sino sobre el significado que se les atribuye" (Groppo, 2002, p. 192), sobre qué voces se habilitan, cuándo y cómo se recuerda y se olvida.

Nuestro interés se centra precisamente en los procesos de selección de información inherentes a los sistemas de información en el conflicto colombiano. A pesar del amplio cuerpo de literatura sobre memoria en América Latina (Groppo, 2002; Crenzel, 2011; Lamilla, 2011; Restrepo, 2011; Jelin, 2001), la producción de memoria colectiva a partir de los sistemas de información en estos contextos ha sido poco estudiada desde los Estudios Sociales de Ciencia y Tecnología (ESCT).

Así las cosas, esta investigación se sitúa en ese espacio y en un escenario enmarcado por la implementación de la Ley 975/2005 de Justicia y Paz en Colombia (y de legislación subsecuente). El objetivo fundamental de la Ley 975/2005 se definió como "facilitar los procesos de paz y la reincorporación individual o colectiva a la vida civil de miembros de grupos armados al margen de la ley, garantizando los derechos de las víctimas a la verdad, la justicia y la reparación" (art. 10). El derecho a la reparación ${ }^{10}$ incluyó "la colaboración eficaz para la localización de personas secuestradas o desaparecidas y la localización de los cadáveres de las víctimas" y "la ayuda para identificarlos y volverlos a inhumar según las tradiciones familiares y comunitarias"11.

Nos enfocamos en este último proceso. Entendimos el sistema de información como un lugar donde confluyen prácticas cotidianas de memoria y estudiamos aquellas acciones que conllevan a la identificación y entrega de un cuerpo. Reconstruimos la trayectoria de producción de información en ese proceso e identificamos los filtros que se ponen en práctica al clasificar información para analizarla bajo estándares científicos, puesto que en el esfuerzo por ofrecer respuestas objetivas $y$ científicamente validadas, e identificar quién le hizo qué a quién, la información que no se ajusta a los estándares del sistema de información es invisibilizada y excluida. Sin embargo, también seguimos los rastros

\footnotetext{
${ }^{10}$ Comprende restitución, indemnización, rehabilitación, satisfacción y garantias de no repetición.

${ }^{11}$ Artículo 44. Este tipo de reparación también se incluyó en la Ley 1448/2011 (artículo 139) como medida de satisfacción.
} 
que van quedando excluidos de esta información y que tienen el potencial de ser rescatados; nos preguntamos por las historias que se pueden construir a partir de lo que se incluye y excluye.

\section{El contexto del sistema de información}

Con la promulgación de la Ley 975/2005 (y posteriormente con la Ley 1448/2011) se inició en Colombia un proceso sin precedentes al utilizar, en medio de la guerra, mecanismos concebidos para post-conflictos (Uprimny, Botero, Restrepo, y Saffon, 2006; Amnistía Internacional, 2012). Con la primera se establecieron penas alternativas para miembros de grupos armados organizados al margen de la ley que se desmovilizaran, cesaran las hostilidades y colaboraran efectivamente con la justicia ${ }^{12}$ (Castro, 2008, p. 244). La segunda se centró en los derechos de las víctimas y su reconocimiento -sin importar el perpetrador-, reconoció la existencia de un conflicto armado y definió medidas para los componentes de la reparación, especialmente la restitución de tierras (Uprimny y Sánchez, 2011; Amnistía Internacional, 2012). Incluyó la creación del Centro de Memoria Histórica encargado, entre otras cosas, de documentar hechos victimizantes ${ }^{13}$. El proceso de búsqueda, exhumación, identificación y entrega de cuerpos como componente de la reparación no cambió entre la primera y la segunda Ley.

Desde su promulgación, las dos leyes han generado controversia y críticas por parte de movimientos de víctimas, organizaciones de derechos humanos y académicos nacionales e internacionales (Laplante y Theidon, 2006; Díaz, 2009; Uprimny et al., 2006; Conpes, 2009; Jaramillo-Marín, 2010; Amnistía Internacional, 2011; 2012; Uprimny y Sánchez, 2011). La Ley 975/2005 fue especialmente criticada puesto que la verdad que buscaba establecer correspondia a una verdad judicial centrada en las confesiones individuales de los desmovilizados y no en las voces de las víctimas. Buscaba judicializar individuos sin ofrecer garantías para reconstruir una verdad histórica y social que pudiera dar cuenta de los determinantes estructurales del conflicto para entender mejor las causas, el contexto

\footnotetext{
${ }_{12}$ Artículos 10 y 11 .

${ }^{13}$ Según la Ley, los archivos judiciales podrán ser usados para "fortalecer la memoria histórica" (Artículo 144, parágrafo 1).
} 
y las responsabilidades de los hechos violentos (Jaramillo-Marín, 2010; Laplante y Theidon, 2006; Díaz, 2009). ${ }^{14}$

Adicionalmente, existen problemas técnicos y logísticos en el proceso de búsqueda, exhumación, identificación y entrega de restos (Gómez y Patiño, 2007; Conpes, 2009). Una de las mayores dificultades es la pregunta sobre quién está desaparecido. Los registros están incompletos y desactualizados y no se han implementado mecanismos que faciliten el cruce de información entre entidades. Lo anterior, sumado a las características del conflicto, a la topografia del país y a las estrategias de los grupos armados para disponer de los restos en lugares de dificil acceso, lleva a que no todos los restos encontrados puedan ser identificados; así, la entrega de restos como componente de reparación no siempre se cumple (Gómez y Patiño, 2007; Conpes, 2009).

\section{Consideraciones teóricas}

\section{Sistemas de información y prácticas de memoria}

Partimos de los ESCT que entienden el mundo como un híbrido entre lo social, lo científico y lo tecnológico; donde lo social moldea y es moldeado por lo tecno-científico de manera constitutiva y simultánea (Hughes, 1983; Winner, 1986; Haraway, 1995; Latour, 1993; 2005; Law, 1991; Mol, 2002; M'Charek, 2005; Agamben, 2009). Así, entendemos el sistema de información estudiado como una red compleja de actores, procesos, objetos, formatos, documentos, bases de datos y plataformas que se utilizan o practican rutinariamente con el fin de identificar restos humanos y devolverlos a sus familiares.

Nos aproximamos a este sistema de información desde dos perspectivas principales. En primer lugar, entendemos que las tecnologias de archivo que hacen parte de este sistema (p. ej. listas, carpetas, formatos estandarizados y bases de datos) y las metodologías de indagación científica forense ${ }^{15}$, constituyen prácticas que dejan rastros

\footnotetext{
Sin embargo, la Ley contempla el derecho a la memoria. Artículo 56: "El conocimiento de la historia de las causas, desarrollos y consecuencias de la acción de los grupos armados al margen de la ley deberá ser mantenido mediante procedimientos adecuados, en cumplimiento del deber a la preservación de la memoria histórica que corresponde al Estado".

${ }^{15}$ La experiencia de la práctica forense en América Latina, Europa del Este y África ha sido de gran importancia documentando violaciones a los DD. HH. y construyendo evidencia importante para los procesos judiciales que estas implican (Ferllini 1999; Schmitt 2002; Baraybar y Gasior, 2006;
} 
del pasado de manera cotidiana. Es decir, prácticas a través de las cuales se recuerda y se construye memoria; aquellas que Bowker (2008) llama prácticas de memoria.

Estas prácticas y tecnologías tienen una característica común: están compuestas de clasificaciones; por ejemplo, las categorias socio-demográficas de los formatos y los tipos de lesiones encontradas en los cuerpos (ver Schmitt, 2002). Las clasificaciones son simultáneamente, formas de organizar el mundo y herramientas de la memoria (Bowker y Star, 2000; Lampland y Star, 2009; Bowker, 2008). Como señalamos previamente, "la memoria [...] es forzosamente una selección" (Todorov, 2000, p.13) y el olvido no es un fracaso de la virtud de recordar sino que es un resultado de esa selección; es útil y necesario, sobre todo frente a excesos de información (Connerton, 2008).

Las clasificaciones permiten precisamente olvidar lo particular, por lo cual necesariamente conllevan tomar decisiones sobre qué incluir y qué excluir; sobre qué puede o no ser recordado, qué es útil para el presente y/o el futuro, de qué recuerdos se compondrá la memoria (Groppo, 2002; Lvovich, 2002; Todorov, 2000; Bowker, 2008). Es así que las clasificaciones constituyen filtros de la memoria. El poder de las clasificaciones radica en invisiblilizar esas decisiones y hacerlas parecer como inevitables. En este sentido, clasificar involucra actos y decisiones eminentemente éticos y políticos, además de técnicos y metodológicos (Bowker y Star, 2000; Lampland y Star, 2009).

Sin embargo, eso que ya ha sido ordenado y clasificado constituye "memoria potencial" (Bowker, 2008, p. 30). La información producida a través de diferentes prácticas y almacenada de manera dispersa ya clasificada "puede ser y será re-ensamblada para tomar la forma de hechos del mundo" (Bowker, 2008, p. 18). Así, las clasificaciones y prácticas de memoria también tienen poder sobre "lo que potencialmente se puede saber"16. Esta memoria potencial se puede relacionar con las huellas del pasado de las que habla Jelin; pueden ser evocadas y rescatadas y una vez superadas las dificultades para acceder a ellas, pueden sacar a la luz elementos del pasado que eran invisibles (2001, p. 11).

Ahora bien, al estar enmarcadas dentro de prácticas científicas entendidas como neutrales, objetivas y ciertas (Haraway, 2004), las tecnologías y sistemas de información construyen una memoria que es

Morgan 2011)

${ }^{16}$ Un ejemplo de este potencial es la reconstrucción que de la caída del régimen egipcio hizo Nadia Idle Alex Nunns en su libro Tweets from Tahir: Egypt's Revolution as it Unfolded, in the Words of the People who Made it. 
verdad (Bowker, 2008, p.4) ${ }^{17}$. En otras palabras, una de las contingencias de las prácticas de la memoria en las ciencias y en los sistemas de información presentados aquí, es que apelan al monopolio de la memoria colectiva, mientras que desde los estudios sobre memoria ha quedado claro que la construcción de memoria colectiva es eminentemente multivocal (Groppo, 2002; Jelin, 2001).

\section{Consideraciones metodológicas}

Estudiamos cualitativamente uno de los laboratorios de identificación especializada de la Fiscalía General de la Nación, pues es a estos laboratorios a donde llegan los cuerpos en reducción esquelética que se deben identificar para ser entregados a sus familias. Concebimos el laboratorio como un escenario cotidiano en donde se reconstruye el pasado (Castillejo, 2007) y se producen verdades particulares acerca del conflicto (que refieren al qué, cómo, dónde, a quién y por quién de la violencia). Es uno de los lugares en donde la violencia se vuelve tangible, medible, fijable y fotografiable. Es también el lugar en donde el cuerpo y sus marcas se vuelven evidencia. Los procesos, objetos, bases de datos y estándares que se utilizan o practican rutinariamente allí son elementos del sistema de información, que permiten la identificación y devolución de restos humanos. En el laboratorio el(los) cuerpo(s) y su(s) historia(s) pueden ser reconstruidos y leídos, como el principal objeto de estudio y fuente de reparación.

En el laboratorio entrevistamos a peritos de antropología, medicina y odontología forense, al coordinador y a la asistente administrativa. De manera complementaria analizamos las unidades documentales (UD) en donde se consigna toda la información de un caso en el laboratorio. Estas incluyen datos sobre la fosa y el cuerpo, información sobre el individuo y el proceso, informes, procedimientos e intervenciones realizadas (por ejemplo interconsultas con laboratorios como balística, grafología o genética) y todas las comunicaciones que se tienen con el(la) Fiscal. Así seguimos la trayectoria de la información habilitada, producida y almacenada en el laboratorio en las unidades documentales, en las prácticas y en las narraciones de las personas entrevistadas. También entrevistamos a Fiscales de la Unidad Nacional de Justicia y Paz (UNJYP), a sus

${ }_{17}$ Los métodos de indagación científica son performativos: al usarlos para medir, clasificar y organizar el mundo, estamos a la vez construyendo un mundo que responde a esos parámetros (Law y Urry, 2004). 
asistentes e investigadores(as), con el fin de entender qué pasaba con y cómo se manejaba la información producida en el laboratorio.

Nuestro trabajo de campo fue multi-situado (Marcus, 1995): buscamos entender y dar cuenta de cómo la información es múltiple (Mol, 2002) y toma diferentes formas, matices y jerarquías según el momento en el que aparece y en el que es requerida. Nos enfocamos en estudiar cómo la información era enactuada ${ }^{18}$ por diferentes actores en diferentes momentos y cómo esta produce una historia particular del conflicto.

\section{Resultados}

La información producida durante el proceso que va de la exhumación de un cuerpo a su potencial identificación y entrega es altamente heterogénea. Comprende procesos y actividades administrativas o técnico-científicas, como el envío de órdenes de exhumación o exámenes periciales; interacciones entre actores como intercambio de llamadas, correos u oficios; resultados de investigación y procesos especializados que incluyen la individualización e identificación de restos, lesiones, causas y maneras de muerte; datos sobre la identidad del sujeto y de su historia social, de violencia y muerte como información socio-demográfica, pertenencia a grupos sociales de diversa índole, circunstancias de la desaparición y relaciones sociales; e información sobre diferentes actores del conflicto, por ejemplo áreas de influencia o estrategias de inhumación de grupos armados.

Esta información es producida en la interacción de múltiples actores que configuran diversas prácticas de memoria: postulados ${ }^{19}$, familiares, peritos, investigadores(as), funcionarios(as) administrativos, procesos judiciales y técnico-científicos, cuerpos, lugares, objetos, bases de datos y documentos. En consecuencia, la información deja rastros diversos y difusos a medida que se registra y almacena de formas igualmente heterogéneas: narrada o codificada, abierta o categorizada, en formatos estandarizados, diarios de campo, expedientes, informes,

\footnotetext{
18 Enactuar es una traducción literal del verbo en inglés To enact. Lo usamos de acuerdo a Mol (2002, p. 41) que refiere la puesta en práctica de objetos-sujetos en contextos particulares. Se emplea esta palabra precisamente para alejarse de otros términos como performar que denotan otros significados. Por eso se utiliza en cursiva.

19 "Miembros del grupo armado organizado al margen de la ley cuyos nombres someta el Gobierno Nacional a consideración de la Fiscalía General de la Nación, que se acojan en forma expresa al procedimiento y beneficios de la Ley 975/2005” (Artículo 17).
} 
fotografias, dibujos, actas, oficios, tablas, bases de datos, plataformas e incluso en la experiencia y en los cuerpos de los diferentes actores.

Esta información diversa transita por el sistema de información a medida que avanza el proceso, permitiendo la comunicación entre actores y posibilitando (o no) la identificación y entrega de cuerpos. Con base en las perspectivas teóricas que informan este estudio, a continuación describimos estas trayectorias y señalamos los filtros de información y los rastros que van quedando en el sistema.

\section{Trayectorias de la información}

Al obtener información sobre la ubicación de una fosa (o fosas) en donde han sido enterradas una o varias personas, un(a) fiscal envía al coordinador del laboratorio una orden de exhumación con datos del lugar y adjunta documentos relevantes. La información aquí puede ser escasa (si no existen documentos previos) o ampliamente detallada (si hay investigaciones anteriores). Por ejemplo, el Formato nacional para búsqueda de personas desaparecidas y el Registro de hechos atribuibles a grupos armados organizados al margen de la ley incluyen desde datos sociodemográficos, información financiera, pertenencia grupal, antecedentes judiciales y descripciones morfológicas de la víctima (registrados en categorias), hasta descripciones de la desaparición con citas textuales. También pueden incluir fichas de campo de investigadores con entrevistas, revisiones documentales o testimonios de postulados a quienes se les solicitan "narraciones claras y precisas de los hechos".

[...] ¿de qué se compone un informe de campo?, en primer lugar lo que narra el postulado, entonces el postulado en una entrevista dice "en B., en el punto llamado J., ahí matamos una niña que era de tez trigueña, bajita”. Ellos no tienen nombres, pero sí van dando algunas características, ahí va la función del investigador, de sacarles todo lo que se pueda. [...] luego se buscan antecedentes de la víctima, el lugar de los hechos [...] En la investigación se pregunta quién ordenó esa ejecución; la mayoría dicen que porque eran guerrilleros, pero entonces cuando tú empiezas a taladrar en el cerebro de los postulados... imagínate a donde va a ir eso, así se va abriendo, eso se vuelve un monstruo, entonces toca manejar son unos procesos grandísimos. (Entrevistado(a) 1) 
El coordinador asigna el caso a un grupo de peritos en antropología, fotografia, topografía y odontología. La exhumación implica un alto grado de incertidumbre y puede tomar varias semanas, pues las condiciones del terreno, de la zona y de la información disponible pueden dificultar encontrar el lugar:

A veces uno llega y llueve por dias, entonces no se puede excavar, o el postulado no se acuerda bien dónde es la tumba, porque eso no es que le den una dirección exacta sino que le dicen "a tantos pasos del palo de mango, de no sé donde”, buscar así es muy difícil. (Entrevistado(a) 2)

Durante la exhumación el grupo de peritos clasifica la información: toma fotografias y elabora mapas y por medio de dibujos y notas describe detalladamente terreno, clima, vegetación, tipo de enterramiento, estado de la fosa y sus características y posición y estado de cuerpos y objetos. Estas prácticas se realizan para fijar la evidencia: "Lo que no recojas en escena no lo puedes reconstruir. Hay que fijar la evidencia, tomar fotografias" (Entrevistado(a) 2).

La información se pasa a un formato que contiene información general, una descripción narrativa del lugar (características del terreno, vegetación, fauna asociada), los procedimientos técnicos utilizados (técnicas arqueológicas de exposición de contexto funerario, método estratigráfico y de pedestal), la clasificación del enterramiento en individual, primario, inalterado, diacrónico, múltiple, sincrónico; el número de individuos, la posición del cuerpo, su grado de descomposición, posición y orientación y un inventario preliminar de restos óseos (cantidad y observaciones). Incluye las observaciones correspondientes: "se encontró sin prendas. En excavación se fracturó el cráneo" (UD\# 1). También incluye "el hallazgo de los signos y elementos de violencia evidentes asociados" clasificados en signos de violencia sexual, signos de tortura y violencia fisica. Por último se describen las prendas y elementos asociados (joyas, documentos, títulos valores).

Estas clasificaciones constituyen memoria potencial: interpretadas por peritos, pueden dar cuenta de las prácticas características de los diferentes actores armados. Por ejemplo, los paramilitares suelen cavar fosas superficiales cerca a las carreteras y descuartizar los cuerpos. Estos datos pueden contribuir en estudios históricos o sociológicos posteriores. Sin embargo, estos análisis se filtran en la primera parte del proceso porque no tienen lugar en los formatos existentes. 
Sus rastros quedan incorporados en el conocimiento experiencial de los(as) peritos:

La información arqueológica es muy rica. Con la información de campo se podría hacer arqueología documental. Pero no podemos, no tenemos tiempo [...] debería haber historiadores, sociólogos, antropólogos. El compromiso era la verdad. (Entrevistado(a) 3)

Asimismo, durante las exhumaciones, los(as) peritos hacen interrogaciones al postulado para dirigir la búsqueda; en ocasiones deben confrontar sus respuestas con los hallazgos del campo (cuando no coinciden). Un(a) de los(as) investigadores nos explicó:

Entonces en la parte de descripción de cómo se encontraron los restos, las fosas, uno se da cuenta. Por ejemplo en el caso de las mujeres, que los interiores están por un lado, el brassier está roto en la parte delantera. Pero en el dictamen no hay inferencias.[...] En las versiones libres, el postulado admite torturas, asesinatos, masacres, pero una violación es muy difícil que la admita. Por ejemplo en esa exhumación [en la que se hallaron prendas rotas] el postulado dice que no la violaron. Al encontrar el cuerpo lo confrontamos [...] Podemos fijar esa información, describiéndola, tomando fotos. El contexto arqueológico solo se ve una vez. Solo hay una oportunidad para verlo. (Entrevistado(a) 6)

Pero estas conversaciones, silencios, confrontaciones y la información que producen, no quedan necesariamente registrados detalladamente o interpretados en los formatos de campo, dado que estos tampoco los requieren. Es decir, en este proceso opera otro filtro, dado, en primer lugar, por la información que solicitan los formatos:

Yo le pregunto informalmente todo: a quién, posible identidad, hechos, profundidad, de qué forma los enterraron, si están completos o por partes. Pero en el informe básicamente son descripciones. Dónde es, la zona, cómo es la vegetación, topografia, posición de los cuerpos, elementos asociados, amarres, prendas sobrepuestas o al lado. Básicamente todo es descripción. (Entrevistado(a)2)

Hay formatos para todo. Lo que no hay, hay que adaptarlo, machetear y acomodar a los sistemas. El sistema está más para el lado 
investigativo que el científico [...] Los de [gestión de] calidad son los que deciden cómo van ciertos formatos, se deben ajustar al que usan todos los grupos. Logramos meter las fotos. (Entrevistado(a)3)

En segundo lugar, el filtro opera a través de los métodos disponibles. En el caso que describe el/la entrevistado(a) 6, debido a que los restos óseos no contienen evidencia que apunte específica e inequívocamente a violencia sexual, los métodos utilizados no pueden producir este dato ${ }^{20}$ :

Con la evidencia al momento de la exhumación se describe pero no se dice qué sugiere. El antropólogo hace la descripción de los hallazgos pero es al fiscal al que le corresponde documentar e investigar eso. (Entrevistado(a) 5)

En este caso puntual, los rastros contenidos en la ficha y en el diario de campo se retoman solo en el caso que un(a) fiscal decida confrontar a un postulado. Sin embargo, la utilidad de registrar esta información está en verificar si los postulados se adhieren a las condiciones de la Ley de decir la verdad y no en investigarla e imputarla como un delito en sí mismo ${ }^{21}$ :

Con esa información se confronta al postulado. [...] Sirve para hacer imputación correcta. Es obligación del postulado decir la verdad. El delito sexual no modifica la pena alternativa pero sí la principal. (Entrevistado(a) 7)

Así, en el caso descrito, aunque la manera como se produce y documenta inicialmente la información permitiría la construcción de historias sobre violencia sexual, la información que atraviesa los filtros de los formatos y los métodos es, en términos de Law y Urry (2004), una en la que la violencia sexual no se hace visible:

\footnotetext{
${ }^{20}$ Aun cuando hay guías que describen qué hallazgos se consideran indicios de violencia sexual y recomiendan su documentación durante los procedimientos forenses (Caicedo, Buenahora y Benjumea, 2009)

${ }^{21}$ A pesar de los esfuerzos por visibilizarlos y de ser considerados una infracción al DIH, y en ocasiones delitos de lesa humanidad o crimenes de guerra, los delitos sexuales no constituyeron un obstáculo para otorgar beneficios en la Ley 975/2005 (Caicedo et al., 2009).
} 
Dentro de Justicia y Paz creo que no se ha tenido en cuenta el delito sexual. Todo depende del fiscal. Con la descripción de campo evidente, con fotografias anexas, del delito sexual, o de la tortura, el fiscal decide si le imputa esos crímenes también. Los delitos sexuales casi nunca se toman en cuenta" [...] En Justicia y Paz por regla los postulados niegan delitos sexuales [...] El delito sexual no es sujeto de rebaja [...] Ese delito se está quedando en el aire. Creo que ha sido la pelea de las ONGs.(Entrevistado(a) 5) ${ }^{22}$

En el laboratorio el coordinador asigna el caso a un grupo de peritos en antropología, medicina forense y odontología. Posteriormente se lavan los restos, la ropa y otros objetos que puedan aportar a la identificación. Todo es fotografiado. Se individualizan los restos para establecer el número mínimo de individuos presentes. El cuerpo se domestica para ser analizado. La violencia se mide, dibuja, toca, fija, fotografia y compara con estándares científicos. Es decir, se vuelve objeto de diversas prácticas de memoria. La información principal que se produce se deriva de un proceso de clasificación de los hallazgos en la cuarteta básica (sexo, edad, altura, raza), la lateralidad y la causa y manera de muerte. La información que no se extraiga de estos métodos forenses no se incluye al no poder ser sustentada y validada científicamente. En este proceso se consultan otros expertos; por ejemplo balística, química o fotografia y bases de datos o sistemas que puedan aportar a la identificación. Cada perito produce un informe usando un formato estandarizado que pide información puntual que solo refiere a su experticia.

Un ejemplo de los filtros que operan en este proceso está en una de las UD analizadas. En las observaciones finales de la ficha de campo se registraron como evidencia física 21 condones, un cuerpo con ropa y calzado de mujer y los documentos de identidad de dos hombres. El(la) antropólogo(a) consignó que el postulado "manifestó que los documentos que se encontraban en el morral [hallado en la tumba] pueden corresponder a un cliente de Leidy ${ }^{23}$ [de quien se sospecha es el cuerpo hallado], que era trabajadora sexual y cuando [el cliente] no tenía plata, dejaba muchas veces como garantía los documentos" (UD\#2). Los restos encontrados estaban atados con sogas y nudos en las muñecas y pies, lo que el(la) perito clasificó como indicios de tortura.

\footnotetext{
${ }_{22}$ Invisibilidad que también se hace evidente en las estadisticas: http://www.fiscalia.gov.co/jyp/ unidad-de-justicia-y-paz/

23 Seudónimo.
} 
Sin embargo, en los subsecuentes análisis forenses, los restos de la posible Leidy presentaron rasgos morfológicos que, según los estándares científicos, correspondían a un esqueleto masculino. A partir de este punto, la evidencia hallada en la fosa y el testimonio del postulado que hacian referencia a una trabajadora sexual fueron dejados de lado. La investigación se enfocó en encontrar la identidad de este hombre y determinar, por medio de análisis genéticos y forenses, si los restos pertenecían a alguno de los dos hombres cuyas cédulas de ciudadanía estaban en la tumba. Finalmente se concluyó que sí y los restos fueron identificados como Jaime ${ }^{24}$ y entregados a sus familiares.

En este caso, el filtro opera a través de los métodos y las clasificaciones a las que son sometidos los cuerpos. Si bien la información registrada en la ficha de campo apuntaba a, al menos un tipo específico de violencia (de género), esta información se quedó detenida en los estándares; la historia que se reconstruyó sobre ese cuerpo estaba basada en el estándar forense del sexo. Una historia basada en la evidencia producida y validada por un método que no puede ver un cuerpo transgresor (que puede ser el de una trabajadora sexual transgenerista) que no cabe ni se concibe en los estándares científicos y sociales que establecen qué es posible contar. Al final, Leidy no existe como víctima ni su caso forma parte de la historia de violencia de género en Colombia; su historia como individuo se interrumpe al ser sometida a los estándares disponibles para su identificación y surge la de un Jaime, del que se sabe que murió y apareció en esa fosa. A menos que se reconstruyan los rastros que quedaron en el sistema (en la ficha de campo), es como si la otra parte de la historia no existiera.

Concluido el estudio, el líder del grupo integra los informes en un informe final estandarizado. Este formato indica que se diligencie "información precisa y concisa". Es decir, en este punto opera otro proceso de selección y por lo tanto otro filtro. Las observaciones y la información detallada, minuciosa, narrada, que habla del contexto de la violencia, de la historia social de víctimas y perpetradores, de los tipos de violencia y de víctimas y de las prácticas caracteristicas de los actores armados, debe volverse concreta y puntual para dar cuenta únicamente de información técnica, interpretación y resumen de los principales hallazgos. Se resume en una tabla final que contiene: cuarteta básica, lateralidad, causa y manera de muerte y características individualizantes.

\footnotetext{
${ }^{24}$ Seudónimo.
} 
En los casos en los que es posible relacionar los restos con un individuo específico, se selecciona un hueso para enviar al laboratorio de genética. Alli se analizan los restos y se comparan con muestras de un familiar ${ }^{25}$. Una vez identificado, el(la) médico(a) forense llena el certificado de defunción que incluye información demográfica y fecha, causa y manera de muerte. La fecha de muerte que se ingresa es la fecha de la desaparición.

En este punto opera otro filtro. La historia de violencia materializada y documentada en los procesos de investigación, búsqueda, exhumación e identificación previamente documentados en detalle, se aplana, compacta, simplifica. Es innegable la necesidad de filtrar información en este punto. Lo que consideramos relevante es que el formato utilizado no corresponde con el contexto en el que se usa, en el que la muerte y la desaparición ocurren en circunstancias y temporalidades diversas. En este caso, el tiempo y lo que este denota, se comprime.

Luego, el(la) líder del grupo anota en el Sistema de Información Red de Desaparecidos y Cadáveres (SIRDEC) que la persona reportada como desaparecida fue hallada muerta e indica el método de identificación. El coordinador del laboratorio envía al(la) fiscal los restos, el reporte final y un acta de entrega. El filtro en este caso tiene que ver con la invisibilización del proceso de identificación. Un proceso complejo, dificil, lento y minucioso que habla del contexto en el que se lleva a cabo (en medio de un conflicto que data de décadas, con múltiples actores con estrategias para desaparecer, inhumar e infundir terror, ante números incontables de víctimas, con limitaciones y riesgos de acceso a las zonas de exhumaciones, entre otros), se vuelve invisible, inmediato, fácil incluso; como en las series de televisión ${ }^{26}$. La complejidad, heterogeneidad y multiplicidad -el monstruo del que habla el/la primer entrevistado(a)- se simplifican en un asunto puntual y/o binario: se identificó, quién era, causa y manera de muerte.

Posteriormente la Subunidad de exhumaciones de la UNJYP programa la fecha de la ceremonia en la cual entrega a la familia un cofre con los restos, un acta, el certificado de defunción y una foto de la persona en vida. Se les pregunta si desean ver los restos. En caso afirmativo, un(a) perito resuelve sus inquietudes y re-construye la historia de lo ocurrido; suele decidir qué es apropiado contar. Es decir,

\footnotetext{
${ }^{25}$ La identificación puede hacerse utilizando la carta dental, pero dada la escasa disponibilidad de información ante mortem y el estado de los restos, esto no es frecuente.

${ }^{26}$ Esta representación de procesos científicos forenses ha sido llamada efecto CSI (Kruse, 2010).
} 
filtra la información que considera inapropiada para una situación muy delicada y sensible:

La familia siempre quiere saber si la persona sufrió y uno les dice lo que sabe pero les da pocos detalles porque para ellos puede ser muy dificil. También a veces les cuesta creer que esa sí sea la persona desaparecida y hacen preguntas sobre el cuerpo [...] y entonces uno les muestra directamente [...] así los ayuda a aceptar la muerte. (Entrevistado(a) 4)

Sin embargo, puede decirse que este es uno de los pocos momentos (si no el único) en el que los familiares son informados de lo que ocurrió (exceptuando las audiencias, que pueden o no suministrar esta información). Como sucede en las etapas ya descritas, lo que cuenta como información válida para contar a los familiares es aquello que es comprobable y medible. Como dijo un(a) entrevistado(a): "En un dictamen no puede haber suposiciones o inferencias que no puedan demostrarse" (Entrevistado(a) 5). Es así que hay elementos que se invisibilizan y quedan como rastros.

\section{Conclusión}

En esta investigación nos preguntamos por el tipo de memoria del conflicto que se construye en Colombia a través del sistema de información disponible para la búsqueda, exhumación, identificación y entrega de restos como componente de reparación.

Mostramos cómo las prácticas de memoria jurídico-científica son, al igual que otras prácticas de memoria, selecciones sobre qué olvidar y qué recordar. Como señalan Groppo (2002), Jelin (2001) y otros, no hay una memoria definitiva, finita, única. La memoria -en este caso la memoria colectiva- está en permanente reconstrucción y a medida que el tiempo avanza, las diferentes versiones del pasado se revisan, afirman, debaten o refutan. Sin embargo, dado el contexto jurídico-científico en el que se construye una memoria colectiva particular sobre el conflicto, encontramos que, como Bowker (2008) señala, esta tiene carácter de verdad al ser presentada como un hecho científico.

Es así como la política de memoria colectiva enmarcada bajo la Ley 975/2005 tiende a invisibilizar la violencia sexual, y los cuerpos transgresores, así como el conocimiento sobre prácticas características 
de grupos armados y la información compleja y heterogénea, pues estas no encajan en los diferentes filtros que encarnan los formatos del sistema. Esto es: no se ha creado un estándar que permita registrar los indicios (de violencia sexual) de manera que no dependan de una interpretación y búsqueda posteriores y que además hagan parte de la información que llega al final del proceso.

Tampoco existen maneras de leer los restos óseos en formas que no sean en términos excluyentes de femenino-masculino. Lo que implica que estas violencias (e historias) no solo se convierten en rastros, sino que no son consideradas tan relevantes como otras. Esto ocurre sobre todo, porque el método utilizado no puede lidiar con información que no queda inequivocamente expuesta en los restos óseos y deja de lado información que si bien podría no encajar en los estándares jurídicos de evidencia, es esencial en la construcción de memoria(s) colectivas del conflicto que vayan más allá de la verdad judicial. En el caso de la violencia sexual o de género por ejemplo, no permiten que se re-configure una memoria colectiva matizada y múltiple en la que estas violencias adquieran una transcendencia pública (Latour, 1993), más allá de ser eventos aislados o de la esfera privada. Lo que podríamos reconfigurar con la información así clasificada es una versión para la cual la violencia sexual y de género o bien son irrelevantes, inexistentes o inconvenientes para un presente aún en medio del conflicto; o peor aún, toleradas.

Ahora bien, también mostramos que es posible rastrear la información producida y encontrar matices que configuren otras historias y permitan sacar a la superficie algunas complejidades que de otra manera, el sistema de información no permitiria contar. En ese sentido, los casos presentados son ejemplos de memoria potencial: de las posibilidades de reconstruir las huellas del pasado (Jelin, 2001) a medida que la información se transforma (Bowker, 2008). Sin embargo, es importante tener en cuenta que estas reconstrucciones también son problemáticas y contingentes. Dadas las limitaciones del campo y de los estándares del sistema, la re-construcción de estos tipos de violencia queda sujeta a que se busque activamente reconstruirla. Sin embargo y precisamente debido a su incertidumbre, se vuelve fundamental tener herramientas para hacerlo: el pasado y la memoria son inherentemente ambiguos, inciertos y están en constante re-configuración (Todorov, 2000).

Teniendo lo anterior en mente, se hace necesario pensar en formular métodos y formatos nuevos que nos permitan enactuar el mundo de 
formas que sean múltiples y matizadas (Law y Urry, 2004), en las que la incertidumbre no se filtre, sino que forme parte de la indagación misma, como fuente fundamental de información relevante y válida en los cuerpos exhumados e identificados. Es decir, abogamos por la búsqueda de estándares en el sistema que, como prácticas de memoria, puedan dar cuenta de la ambigüedad (en lugar de invisibilizarla) aunque estén simultáneamente alineados con métodos jurídico-científicos. Hacemos un llamado a buscar maneras de estar con la incertidumbre (Puig de la Bellacasa, 2011, Pérez-Bustos, Olarte-Sierra y Díaz del Castillo, 2014), de forma que la memoria colectiva así producida no refuerce la invisibilidad de por ejemplo, la violencia sexual y de género en el conflicto en Colombia y que tampoco se asemeje a una forma de tolerancia.

\section{Referencias}

Agamben, G. (2009). What Is An Apparatus? Stanford: Stanford University Press. Amnistía Internacional (2011). Informe anual 2011. El estado de los derechos en el mundo. Colombia. Disponible en: http://www.amnesty.org/es/region/ colombia/report-2011\#section-32-6.

Amnistía Internacional (2012). Colombia: Ley de víctimas y restitución de tierras. Análisis de Amnistía Internacional. Londres: Amnesty International Publications.

Ball, P., Spirer, H. y Spirer L. (2000). Making the Case: Investigating large scale human rights violations using information systems and data analysis. Washington, DC: American Association for the Advancement of Science.

Baraybar J.P. y Gasior, M. (2006). Forensic Anthropology and the most probable cause of death in cases of violations against International Humanitarian Law: An example from Bosnia and Herzegovina. Journal of forensic Sciences, 51, 103-108.

Bowker, G. C. (2008). Memory practices in the sciences. Cambridge, Massachusetts: The MIT Press.

Bowker, G. C. y Star, S. L. (2000). Sorting things out: Classification and its consequences. Cambridge, Massachusetts: The MIT Press.

Caicedo, L.P., Buenahora, N. y Benjumea, N. (2009). Guía para llevar casos de violencia sexual. Propuestas de argumentación para enjuiciar crimenes de violencia sexual cometidos en el marco del conflicto armado colombiano. Bogotá: Corporación Humanas - Centro Regional de Derechos Humanos y Justicia de Género.

Castillejo, A. (2007). La globalización del testimonio. Historia, silencio endémico y los usos de la palabra. Antípoda, 4, 76-99. 
Castro, A. H. (2008). Paramilitarismo, desmovilización y reinserción: la ley de justicia y paz y sus implicaciones en la cultura politica, la ciudadanía y la democracia en Colombia. Bogotá: UNIJUS.

Conpes (2009). Consolidación de los mecanismos de búsqueda e identificación de personas desaparecidas en Colombia. Documento Conpes 3590. Bogotá: Departamento Nacional de Planeación.

Connerton, P. (2008). Seven Types of forgetting. Memory Studies. (1), 59

Crenzel, E. (2011). La transmisión interrogada. Jóvenes conocimiento y memoria de la represión en el Hospital Posadas, Buenos Aires, Argentina. Universitas Humanistica, 72 (72), 111-132.

Díaz, I. (2009). El rostro de los invisibles. Víctimas y su derecho a la verdad, justicia, reparación y no repetición. En: López, X., Muñoz, M. R. y Bondia García, D. Victimas Invisibles, Conflicto Armado y Resistencia Civil En Colombia (pp.17-35). Barcelona: Huygens Editorial.

Ferllini, R. (1999). The role of anthropology in human rights issues. En Fairgrieve S. (Ed.). Forensic Osteological Analysis: A book of case studies (pp.63-85). Sprinfield, IL.; Charles C Thomas.

Gómez, A. M. y Patiño, A. (2007). Who is missing? Problems in the application of forensic archaeology and anthropology in Colombia's conflict. En Ferllini, R. (Ed). Forensic Archaeology and Human Rights Violations (pp. 170-204). Springfield: Charles C Thomas Publisher.

Groppo, B. (2002). Las politicas de la memoria. Sociohistórica, (11-12). Disponible en: http://www.fuentesmemoria.fahce.unlp.edu.ar/art_revistas/pr.3067/pr. 3067.pdf

Halbwachs, M. y Namer, G. (1994). Los marcos sociales de la memoria. Barcelona: Anthropos Editorial.

Haraway, D. (1995). Ciencia, cyborgs y mujeres. La reinvención de la naturaleza. Madrid: Ediciones Cátedra.

Haraway, D. (2004). Testigo_Modesto@Segundo_Milenio. HombreHembra@_ Conoce_Oncoratón ${ }^{\circledR}$. Barcelona: Editorial UOC.

Hughes, T. (1983). Networks of Power. Electrification in Western Society 18801930. Baltimore y Londres: The Johns Hopkins University Press.

Huridocs. (s.f.) Disponible en: www.huridocs.org.

Jaramillo-Marín, J. (2010). Reflexiones sobre los “usos” y “abusos" de la verdad, la justicia y la reparación en el proceso de Justicia y Paz colombiano (20052010). Papel Político, 15 (1), 13-46.

Jelin, E. (2001). ¿De qué hablamos cuando hablamos de memoria? En Los trabajos de la memoria. España: Siglo Veintiuno editores, 2001. Disponible en: http://www.cholonautas.edu.pe/modulo/upload/JelinCap2.pdf 
Knuth, R. (2006). Sovereignty, Globalism, and Information Flow in Complex Emergencies. The Information Society, 15 (1), 11-19.

Kruse, C. (2010). Producing absolute truth: CSI science as wishful thinking. American Anthropologist, 112 (1), 79-91.

Lamilla, E. (2011). El cementerio central de Neiva (Huila): Escenario de activación, reinterpretación y disputa de múltiples memorias. Universitas Humanistica, 72 (72), 180-210.

Lampland, M. y Star, S. L. (2009). Standards and their Stories. How Quantifying, Classifying, and Formalizing Practices Shape Everyday Life. Ithaca y Londres: Cornell University Press.

Laplante, L. y Theidon, K. (2006). Transitional Justice in Times of Conflict: Colombia's Ley de Justicia y Paz. Michigan Journal of International Law, 28 (1), 49-108.

Latour, B. (1993). We have never been modern. Londres: Harvester Wheatsheaf.

Latour, B. (2005). Reassembling the social. Oxford: Oxord University Press.

Law, J. (1991). Introduction: monsters, machines and sociotechnical relations. En Law, J. (Ed.). A sociology of monsters. Essays on power, technology and dominations (pp. 1-25). Londres y NuevaYork: Routledge.

Law, J. y Urry, J. (2004). Enacting the Social. Economy and Society, 33 (3), 339-410.

Lvovich, D. (2002). [Reseña sobre] Groppo, Bruno y Flier, Patricia, (2001). La imposibilidad del olvido. Recorridos de la memoria en Argentina, Chile y Uruguay, La Plata. Al Margen/ Bibliothéque de Documentation Internationale Contemporaine. Sociohistórica, (11-12). Disponible en: http://www.fuentesmemoria.fahce.unlp. edu.ar/art_revistas/pr.3827/pr.3827.pdf

Marcus, G. E. (1995). Ethnography in/of the world system: The emergence of Multi-sited Ethnography, Annual Review of Anthropology, 24 (1), 95-117.

M'charek, A. (2005). Human Genome Diversity Project. An ethnography of Scientific practice. Cambridge: Cambridge University Press.

Mol, A. (2002). The body multiple. Ontology in medical practice. Durham: Duke University Press.

Morgan, J. M. (2011). Proving Genocide: Forensic Anthropologist's Role In Developing Evidence To Convict Those Responsible For Genocide Electronic Theses, Treatises and Dissertations. Paper 5053.

Pérez-Bustos, T., Olarte-Sierra, M. F. y Díaz del Castillo A. (2014). Working with care: experiences of invisible women scientists practicing forensic genetics in Colombia. En Beyond imported magic: Studying Science and Technology in Latin America(en prensa). Massachusetts: MIT University Press.

Puig de la Bellacasa, M. (2011). Matters of Care in Technoscience. Assembling Neglected Things. Social Studies of Science, 41 (1), 85-106. 
Restrepo, G. I. (2011). Memoria e historia de la violencia en San Carlos y Apartadó. Universitas Humanística, 72 (72), 157-188.

Schmitt, S. (2002). Mass Graves and the collection of forensic evidence: Genocide, war Crimes, and crimes against humanity. En Haglund, W. D. y Sorg, M. (Eds.). Advances in forensic: method, Theory, and archaeological perspectives (pp. 277-292). Boca Ratón FL: CRC Press LLC.

Steinberg, M., Height, C., Mosher, R. y Bampton, M. (2006).Mapping massacres: GIS and state terror in Guatemala. Geoforum, 37, 62-68.

Todorov, T. (2000). Los abusos de la memoria. Barcelona: Paidós.

Uprimny, R., Botero, C., Restrepo, E. y Saffon, M. (2006). ¿Justicia transicional sin transición? Reflexiones sobre verdad, justicia y reparación en Colombia. Bogotá: Dejusticia. Centro de Estudios de Derecho, Justicia y Sociedad.

Uprimny, R. y Sánchez, N. (2011). Ley de Víctimas: avances, limitaciones y retos. UN periódico 147. Disponible en: http:/ / www.unperiodico.unal.edu.co/ en/dper/article/ley-de-victimas-avances-limitaciones-y-retos.html

Winner, L. (1986). The Whale and The Reactor: A Search for Limits in an Age of High Technology. Chicago: University of Chicago Press.

\section{Cómo citar este artículo}

Olarte-Sierra, M. F., Díaz del Castillo, A., Pulido, N., Cabrera, N. y Suárez, R. (2015). Verdad e incertidumbre en el marco del conflicto en Colombia: una mirada a los sistemas de información como prácticas de memoria. Universitas Humanística, 79, 233-254. http:/ /dx.doi.org/ 10.11144/Javeriana.UH79.vimc 